\title{
Analog Coding for Delay-Limited Applications
}

\author{
Mahmoud Taherzadeh and Amir. K. Khandani \\ Coding \& Signal Transmission Laboratory(www.cst.uwaterloo.ca) \\ Dept. of Elec. and Comp. Eng., University of Waterloo, Waterloo, ON, Canada, N2L 3G1 \\ e-mail: \{taherzad, khandani\}@ cst.uwaterloo.ca, Tel: 519-8848552, Fax: 519-8884338
}

\begin{abstract}
In this paper, we consider the problem of sending an analog source over an additive white Gaussian noise channel. The traditional analog coding schemes suffer from the threshold effect. We introduce two robust schemes for analog conding. Unlike the previous methods, the new methods asymptotically achieve the optimal scaling of the signal-to-distortion-ratio (SDR) without being affected by the threshold effect. Also, we show that approximated versions of these techniques perform well for the practical applications, with a low complexity in encoding/decoding.
\end{abstract}

\section{INTRODUCTION}

In many applications, delay-limited transmission of analog sources over an additive white Gaussian channel is needed. Also, in many cases the exact signal-to-noise ratio is not known at the transmitter, and may vary over a large range of values. Two examples of this scenario are transmitting an analog source over a quasi-static fading channel and/or multicasting it to different users (with different channel gains).

Without considering the delay limitations, digital codes can theoretically achieve the optimal performance. Indeed, for the ergodic channels, Shannon's source-channel coding separation theorem [1] [2] ensures the optimality of separately designing source and channel codes. However, for the case of a limited delay, several articles [3] [4] [5] [6] [7] have shown that joint source-channel codes have a better performance, compared to the separately designed source and channel codes (which are called tandem codes). Also, digital coding is very sensitive to the mismatch in the estimation of the channel signal-to-noise-ratio (SNR).

To avoid the saturation effect of digital coding, in [8] and [9] analog codes, based on dynamical systems are proposed. Although these codes can provide asymptotic gains (for high SNR) over the simple repetition code, they suffer from a threshold effect. Indeed, when the SNR becomes less than a certain threshold, the performance of these systems degrades severely. Therefore, the parameters of these methods should be chosen according to the operating SNR, hence, these methods are still very sensitive to the errors in the estimation of SNR. Also, although the performance of the system is not saturated for the high SNR (unlike digital codes), the scaling of the end-to-end distortion is far from the theoretical bounds.

In this paper, we present two analog coding schemes which have better SNR scalings, and one of them achieves almost the same scaling as the theoretical bound, with just a single mapping for different SNR values. We show that these schemes are not vulnerable to any threshold effect (unlike the previous techniques [8] [9]). Because a single mapping achieves the near-optimum SDR scaling for all ranges of SNR, the proposed technique is robust to the variations of the channel SNR, unlike the other alternatives. This robustness is a very important feature for transmission over channels with unknown SNR.

\section{SYSTEM MODEL AND THEORETICAL LIMITS}

We consider a memoryless $\left\{X_{i}\right\}_{i=1}^{\infty}$ uniform source with zero mean and variance $\frac{1}{12}$, i.e. $-\frac{1}{2} \leq x_{i}<\frac{1}{2}$. For other sources, such as the Gaussian source, we can use the standard companding techniques. Also, the samples of the source sequence are assumed independent with identical distributions (i.i.d.).

The transmitted signal is sent over an additive white Gaussian noise (AWGN) channel. The problem is to map the one-dimensional signal to the $N$-dimensional channel space, such that the effect of the noise is minimized. This means that the data $x,-\frac{1}{2} \leq x<\frac{1}{2}$, is mapped to the transmitted vector $\mathbf{s}=\left(s_{1}, \ldots, s_{N}\right)$. At the receiver side, the received signal is $\mathbf{y}=\mathbf{s}+\mathbf{z}$ where $\mathbf{z}=\left(z_{1}, \ldots, z_{N}\right)$ is the additive white Gaussian noise with variance $\sigma$.

As an upper bound on the performance of the system, we can consider the case of delay-unlimited. In this case, we can use Shannon's theorem on the separation of source and channel coding. By combining the lower bound on the distortion of the quantized signal (using the rate-distortion formula) and the capacity of $N$ parallel Gaussian channels with the noise variance $\sigma^{2}$, we have [9]

$$
D \geq c \sigma^{2 N}
$$

where $c$ is a constant number and $D$ is the average distortion.

\section{CODES BASED ON DYNAMICAL SYSTEMS AND HYBRID DIGITAL-ANALOG CODING}

Previously, two related schemes, based on dynamical systems, have been proposed for the scenario of delay-limited analog coding:

1) Shift-map dynamical system [8]

2) Spherical shift-map dynamical system [9]

\section{A. Shift-map dynamical system}

In [8], an analog transmission scheme based on shift-map dynamical systems is presented. In this method, every analog data $x$ is mapped to the modulated vector $\left(s_{1}, \ldots, s_{N}\right)$ where 


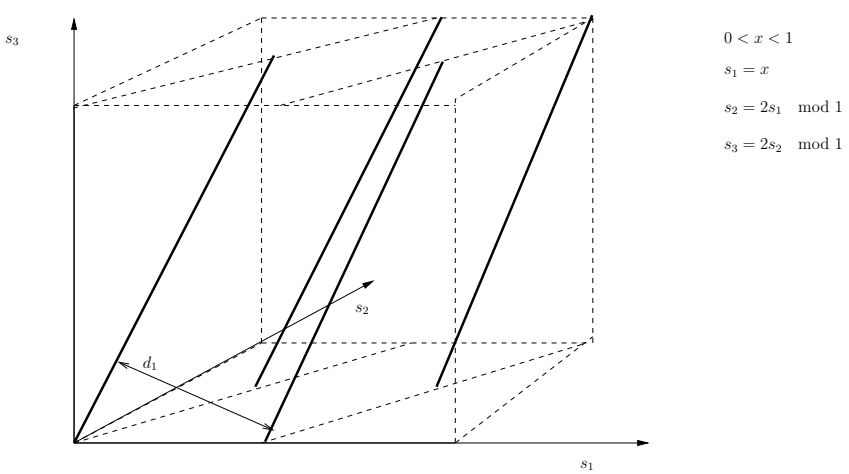

Fig. 1. The shift-map modulated signal set for $N=3$ dimensions and $a=2$.

$$
\begin{gathered}
s_{1}=x \quad \bmod 1 \\
s_{i+1}=b_{i} s_{i} \quad \bmod 1, \quad \text { for } 1 \leq i \leq N-1
\end{gathered}
$$

where $b_{i}$ is an integer number, $b_{i} \geq 2$. The set of modulated signals generated by the shift map consists of $b=b_{1} \ldots b_{N-1}$ parallel segments inside an $N$-dimensional unit hypercube. In [9], the authors have shown that by appropriately choosing the parameters $\left\{b_{i}\right\}$ for different SNR values, one can achieve the SDR scaling (versus the channel SNR) with the slope $N-\epsilon$, for any positive number $\epsilon$. Indeed, we can have these bounds on the end-to-end distortion:

Theorem 1 Consider the the shift-map analog coding system which maps the modulating signals to $N$-dimensional modulated vectors,

i) For any noise variance $\sigma^{2}$, we can find a number a such that for the shift-map scheme with the parameters $b_{i}=a^{i}$, the distortion of the decoded signal $D$ is bounded as

$$
D \leq c \sigma^{2 N}(-\log \sigma)^{N-1}
$$

where $c$ is some constant number (depending on only $N$ ).

ii) For any shift-map scheme, the output distortion is lower bounded as

$$
D \geq c^{\prime} \sigma^{2 N}(-\log \sigma)^{N-1}
$$

where $c^{\prime}$ is a constant number.

\section{B. Spherical shift-map dynamical system}

In [9], a spherical code based on the linear system $\dot{\mathbf{s}}_{T}=\mathbf{A} \mathbf{s}_{T}$ is introduced, where $\mathbf{s}_{T}$ is the $2 N$-dimensional modulated signal and $\mathbf{A}$ is a skew-symmetric matrix, i.e. $\mathbf{A}^{T}=-\mathbf{A}$.

This scheme is very similar to the shift-map scheme. Indeed, with an appropriate change of coordinates, the above modulated signal can be represented as

$$
\begin{gathered}
\mathbf{s}_{T}=\frac{1}{\sqrt{N}}\left(\cos 2 \pi x, \cos 2 a \pi x, \ldots, \cos 2 a^{N-1} \pi x,\right. \\
\left.\sin 2 \pi x, \sin 2 a \pi x, \ldots, \sin 2 a^{N-1} \pi x\right)
\end{gathered}
$$

for some parameter $a$.
If we consider $\mathbf{s}_{s m}$ as the modulated signal generated by the shift-map scheme with parameters $b_{i}=a^{i}$, in (3), then, (3) can be written in the vector form as

$$
\mathbf{s}_{T}=\left(\operatorname{Re}\left\{e^{\pi i \mathbf{s}_{s m}}\right\}, \operatorname{Im}\left\{e^{\pi i \mathbf{s}_{s m}}\right\}\right) .
$$

The relation between the spherical code and the linear shift-map code is very similar to the relation between PSK and PAM modulations. Indeed, the spherical shift-map code and PSK modulation are, respectively, the linear shift-map and PAM modulations which are transformed from the unit interval, $\left[\frac{-1}{2}, \frac{1}{2}\right)$, to the unit circle.

For the performance of the spherical codes, the same result as Theorem 1 is valid. Indeed, for any parameters $a$ and $N$, the spherical code asymptotically has a saving of $\frac{(2 \pi)^{2}}{12}$ or $5.17 \mathrm{~dB}$ in the power. This asymptotic gain results from transforming the unit-interval signal set (with length 1 and power $\frac{1}{12}$ ) to the unit-circle signal set (with length $2 \pi$ and power 1) . However, the spherical code uses $2 N$ dimensions (compared to $N$ dimensions for the linear shift-map scheme).

For both these methods, for any fixed parameter $a$, the output SDR asymptotically has linear scaling with the channel SNR. The asymptotic gain (over the simple repetition code) is proportional to $a^{2(N-1)}$ (because the modulated signal is stretched approximately $a^{N-1}$ times) ${ }^{1}$. Therefore, larger scaling parameters $a$ results in higher asymptotic gains. However, by increasing $a$, the distance between the parallel segments of the modulated signal set decreases. This distance is approximately $\frac{1}{a}$ and for the low SNRs (when the noise variance is larger than or comparable to $\frac{1}{a}$ ), jumping from one segment of the modulated signal set to another one becomes the dominant factor in the distortion of the decoded signal which results in a poor performance in this SNR region. Thus, there is a trade-off between the gain in the high-SNR region and the critical noise level which is fatal for the system. By increasing the scaling parameter $a$, the asymptotic gain increases, but at the same time, a higher SNR threshold is needed to achieve that gain. In [10], the authors have combined the dynamical-system schemes with LDPC and iterative decoding to slightly reduce the critical SNR threshold. However, overall behavior of the output distortion is the same for all these methods.

The shift-map analog coding system can be seen as a slight variation of a Hybrid Digital-Analog (HDA) joint source-channel code. Various types of these hybrid schemes are investigated in [11] and [12]. Indeed, for the shift-map system, we can rotate the modulated signal set such that all the parallel segments of it become aligned in the direction of one of the dimensions. In this case, by slightly changing the support region of the modulated set (which is a rotated $N$-dimensional cube) to the standard cube, we obtain a new similar modulation with almost the same performance. In the new modulation, the information signal is quantized by

\footnotetext{
${ }^{1}$ The exact asymptotic gain is equal to the scaling factor of the signal set, i.e. $a^{2(N-1)}\left(1+\frac{1}{a^{2}}+\ldots+\frac{1}{a^{N-1}}\right)$ for the shift map and $\frac{(2 \pi)^{2}}{12} a^{2(N-1)}\left(1+\frac{1}{a^{2}}+\ldots+\frac{1}{a^{N-1}}\right)$ for the spherical shift map.
} 
$a^{N-1}$ points in an $(N-1)$-dimensional sub-space and the quantization error is sent over the remaining dimension.

Regarding the scaling of the output distorsion, the performance of the shift-map scheme, with appropriate choice of parameters for each SNR, is very close to the theoretical limit. In fact, the output distortion scales as $\sigma^{2 N}(-\log \sigma)^{N-1}$, instead of being proportional to $\sigma^{2 N}$. However, for any fixed set of parameters, the output SNR (versus the input SNR) is saturated by the unit slope (instead of $N$ ). This shortcoming is an inherent drawback of schemes like the shift-map code or spherical code (which are based on dynamical systems). Indeed, in [13], it is shown that no single differentiable mapping can achieve an asymptotic slope better than 1 . This article addresses this shortcoming.

\section{A NEW APPROACH}

We propose two schemes for analog coding using a bandwidth expansion factor of $N$. In both these techniques, the mapping is not differentiable and they can achieve slopes grater than 1 (for the curve of the output SNR). Especially, in the second scheme, a single modulation scheme achieves the optimal slope of $N$. However, in certain ranges of SNR, first scheme can provide a better performance. Although these methods use the infinite binary expansion of real numbers, we will see that practical approximations of these methods still perform well.

\section{A. Scheme I}

For the modulating signal $x,-\frac{1}{2} \leq x<\frac{1}{2}$, we consider the binary expansion of $x+\frac{1}{2}$ :

$$
x+\frac{1}{2}=\left(\overline{0 \cdot b_{1} b_{2} b_{3} \cdots}\right)_{2}
$$

Now, we construct $s_{1}, s_{2}, \ldots, s_{N}$ as

$$
\begin{gathered}
s_{1}=\left(\overline{0 \cdot b_{1} b_{N+1} b_{2 N+1} \cdots}\right)_{\alpha} \\
s_{2}=\left(\overline{0 \cdot b_{2} b_{N+2} b_{2 N+2} \cdots}\right)_{\alpha} \\
s_{N}=\left(\overline{0 \cdot b_{N} b_{2 N} b_{3 N \cdots}}\right)_{\alpha}
\end{gathered}
$$

where $\left(\overline{0 \cdot b_{1} b_{2} b_{3} \cdots}\right)_{\alpha}$ is the base- $\alpha$ expansion ${ }^{2}$.

Theorem 2 In the proposed scheme, for any $\alpha>2$, the output distortion $D$ is upper bounded by

$$
D \leq c \sigma^{2 \beta}(-\log \sigma)^{N}
$$

where $c$ is independent of $\sigma$ and $\beta=N \frac{\log 2}{\log \alpha}$.

Proof: Consider $w_{i}$, as the Gaussian noise on the $i$ th dimension:

\footnotetext{
${ }^{2}$ In this article, we define the base- $\alpha$ expansion, for any real number $\alpha>2$ and any binary sequence $\left(b_{1} b_{2} b_{3} \ldots\right)$, as $\left(\overline{0 \cdot b_{1} b_{2} b_{3} \cdots}\right)_{\alpha} \triangleq$ $\sum_{i=1}^{\infty} a_{i} \alpha^{-i}$.
}

$$
\begin{gathered}
\operatorname{Pr}\left\{\left|w_{i}\right|>2 \sqrt{N} \sigma \sqrt{-\log \sigma}\right\}= \\
Q(2 \sqrt{N} \sqrt{-\log \sigma}) \leq e^{-\frac{4 N(-\log \sigma)}{2}}=e^{-2 N(-\log \sigma)}=\sigma^{2 N}
\end{gathered}
$$

Now, we bound the distortion, conditioned on $\left|w_{i}\right| \leq$ $2 \sqrt{N} \sigma \sqrt{-\log \sigma}$ for $1 \leq i \leq N$ :

If the $k$ th digit of $s_{i}$ and $s_{i}^{\prime}$ are different,

$$
\begin{gathered}
\left|s_{i}-s_{i}^{\prime}\right| \geq \\
\left(\frac{0 \cdot \underbrace{0 \ldots 0}_{k-1} 1000 . .}{}\right)_{\alpha}-(\overline{0 \cdot \underbrace{0 \ldots 0}_{k-1} 0111 \ldots})_{\alpha} \\
>(\alpha-2) \alpha^{-(k+1)}
\end{gathered}
$$

Therefore, if $\left|s_{i}-s_{i}^{\prime}\right| \leq \delta$ for any $\delta>0$, the first $k$ digits of $s_{i}$ and $s_{i}^{\prime}$ are the same, where $k \geq\left\lfloor-\log _{\alpha}\left(\frac{\delta}{\alpha-2}\right)\right\rfloor-1$. Now, by considering $\delta=4 \sqrt{N} \sigma \sqrt{-\log \sigma}$,

$$
\begin{gathered}
\left|s_{i}-s_{i}^{\prime}\right| \leq 2\left|w_{i}\right| \leq 4 \sqrt{N} \sigma \sqrt{-\log \sigma} \\
\Longrightarrow k \geq\left\lfloor-\log _{\alpha}\left(\frac{4 \sqrt{N} \sigma \sqrt{-\log \sigma}}{\alpha-2}\right)\right\rfloor-1
\end{gathered}
$$

Therefore, for $1 \leq i \leq N$, the first

$$
\left\lfloor-\log _{\alpha}\left(\frac{4 \sqrt{N} \sigma \sqrt{-\log \sigma}}{\alpha-2}\right)\right\rfloor-1
$$

digits of $s_{1}, s_{2}, \ldots, s_{N}$ can be decoded without any error, hence, the first

$$
N\left(\left\lfloor-\log _{\alpha}\left(\frac{4 \sqrt{N} \sigma \sqrt{-\log \sigma}}{\alpha-2}\right)\right\rfloor-1\right)
$$

bits of the binary expansion of $x$ can be reconstructed perfectly. In this case, the output distortion is bounded by

$$
\begin{gathered}
\sqrt{D} \leq 2^{-N\left(\left\lfloor-\log _{\alpha}\left(\frac{4 \sqrt{N} \sigma \sqrt{-\log \sigma}}{\alpha-2}\right)\right\rfloor-1\right)} \\
\leq c_{1} \sigma^{\beta}(-\log \sigma)^{\frac{N}{2}}
\end{gathered}
$$

where $c_{1}$ depends only on $\alpha$ and $N$.

By combining the upper bounds for the two cases,

$$
\begin{gathered}
D \leq \operatorname{Pr}\left\{\left|w_{i}\right|>2 \sqrt{N} \sigma \sqrt{-\log \sigma}\right\}+c_{1} \sigma^{\beta}(-\log \sigma)^{\frac{N}{2}} \\
\leq c \sigma^{2 \beta}(-\log \sigma)^{N}
\end{gathered}
$$

According to the theorem 2, for any $\epsilon>0$, we can construct a modulation scheme that achieves the asymptotic slope of $N-\epsilon$ (for the curve of output SNR). As expected (according to the result by Ziv [13]), none of these mappings are differentiable. Indeed, these mappings have 
fractal structures, where the parameter $\beta$, which determines the asymptotic slope of the curve, is the dimension of the fractal. Intuitively, this means that among the $N$ available dimensions, only $\beta$ dimensions are effectively used.

By decreasing $\alpha$, we can increase the asymptotic slope $\beta$. However, it also degrades the low-SNR performance of the system. This phenomenon is observed in figure 1 .

\section{B. Scheme II}

Although by Scheme I we can construct mappings that achieve near-optimum slope for the curve of SDR (versus the channel SNR), none of these mappings can achieve the optimum slope $N$. To achieve the optimum slope with a single mapping, we slightly modify the scheme I:

For the modulating signal $x$, consider $x+\frac{1}{2}=$ $\left(\overline{0 . b_{1} b_{2} b_{3} \ldots}\right)_{2}$. We construct $s_{1}, s_{2}, \ldots, s_{N}$ as

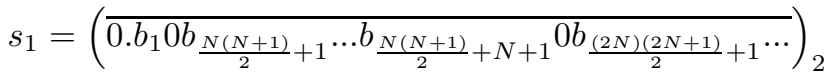

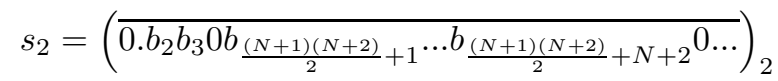

$$
\begin{aligned}
& s_{N}=\left(\frac{\cdots}{0 . b_{\frac{N(N-1)}{2}+1} \cdots b_{\frac{N(N+1)}{2}} 0 \cdots}\right)_{2}
\end{aligned}
$$

The difference between this scheme and Scheme I is that instead of assigning the $k N+i$ th bit to the signal $s_{i}$, the bits of the binary expansion of $x+\frac{1}{2}$ are grouped such that the $l$ th group $(l=k N+i)$ consists of $l$ bits and is assigned to the $i$ th user.

Theorem 3 Using the mapping constructed by Scheme II, the output distortion $D$ is upper bounded by

$$
D \leq c_{1} \sigma^{2 N} 2^{c_{2} \sqrt{-\log \sigma}}
$$

where $c_{1}$ and $c_{2}$ are constant.

Proof: Consider $w_{i}$ as the Gaussian noise on the $i$ th channel and assume that $n$ is selected such that

$$
\sum_{k=1}^{n} k N+i \leq-\log _{2} \sigma<\sum_{k=1}^{n+1} k N+i
$$

The probability that $\left|w_{i}\right| \geq \frac{2^{-\sum_{k=1}^{n-1} k N+i}}{2}$ is negligible. Indeed,

$$
\begin{gathered}
\operatorname{Pr}\left\{\left|w_{i}\right| \geq 2^{\frac{-\sum_{k=1}^{n-1} k N+i}{2}} \mid-\log _{2} \sigma \geq \sum_{k=1}^{n} k N+i\right\} \leq \\
Q\left(2^{n N-1}\right) .
\end{gathered}
$$

On the other hand, when $\left|w_{i}\right|<2^{\frac{-\sum_{k=1}^{n-1} k N+i}{2}}$, the first $\sum_{k=1}^{n-1} k N+i$ bits of $s_{i}$ can be decoded error-freely. The same is true for all $1 \leq i^{\prime} \leq i$, and for $i<i^{\prime} \leq N$, the first $\sum_{k=1}^{n-2} k N+i^{\prime}$ can be decoded error-freely. Thus, the first $\sum_{j=1}^{(n-1) N+i} j$ bits of $x$ can be decoded error-freely. Now,

$$
\begin{gathered}
\sum_{j=1}^{(n-1) N+i} j \geq \\
N \sum_{k=1}^{n-2} k N+i \geq \\
N \sum_{k=1}^{n+1} k N+i-N(n N+i+(n+1) N+i) \geq \\
N \sum_{k=1}^{n+1} k N+i-N^{2}(2 n+3) \geq \\
N \sum_{k=1}^{n+1} k N+i-c_{2} \sqrt{\sum_{k=1}^{n+1} k N+i}
\end{gathered}
$$

where $c_{2}$ depends only on $N$. Therefore, by using the assumption (24),

$$
\begin{gathered}
\sum_{j=1}^{(n-1) N+i} j \geq \\
-N \log _{2} \sigma-c_{2} \sqrt{-\log _{2} \sigma}
\end{gathered}
$$

Therefore the output distortion is bounded by

$$
\begin{gathered}
D \leq 2^{-2 \sum_{j=1}^{(n-1) N+i} j} \\
\leq 2^{2 N \log _{2} \sigma+2 c_{2} \sqrt{-\log _{2} \sigma}} \\
\Longrightarrow D \leq c_{1} \sigma^{2 N} 2^{c_{2} \sqrt{-\log \sigma}} .
\end{gathered}
$$

\section{EXTENSIONS FOR LARGER DELAYS}

In the digital transmission systems, coding over a long block of data can provide a coding gain and improve the performance of the system. Similar improvements can be obtained in analog coding by coding over longer blocks. For example, for a bandwidth expansion of 2, instead of mapping the information signal $x$ to a 2-dimensional vector, we can map a block of length 3 to a 6-dimensional vector. This approach increases the delay of the system; however, it results in a coding gain. Also, in schemes similar to hybrid digital-analog coding (such as shift-map coding scheme), this approach can reduce the required SNR threshold of the system. Therefore, this approach can be usefull when there are only moderate delay constraints.

To elaborate this approach, at first we consider the simple case of the hybrid digital-analog coding with a bandwidth expansion of 2 . In the simple version of this scheme (which is very similar to the shift-map scheme with $N=2$ ), each information signal $x$ is quantized by $a$ points (as the 
transmitted signal $s_{1}$ ) and the the scaled version of the quantization noise is sent over the next dimension (as the transmitted signal $s_{2}$ ). As a natural extention of this scheme for larger blocks of data, we can quantize a block of the information signals (of length $M$ ) by using points from a dense $M$-dimensional lattice. If we use the same number of quantization points, this solution reduces the required SNR threshold by $\gamma_{L}$ where $\gamma_{L}$ is the coding gain of the lattice. Also, it translates the shaping gain of the lattice to the asymptotic performance of the system (in terms of reduction in the output distortion). For example, if we map 3-tuple blocks of data to 6-dimensional vectors by using the quantization based on the lattice $D_{3}$ [14], compared to the simple 1-to-2 scheme, we can reduce the required SNR threshold by about $1 \mathrm{~dB}$ (the coding gain of $D_{3}$ ) and also increase the asymptotic gain of the scheme.

A similar idea can be applied for the schemes proposed in the previous section. Consider $L$ as a binary lattice such that $2^{K} \mathbb{Z}^{M} \subset L \subset \mathbb{Z}^{M}$ and $\left|L / 2^{k} \mathbb{Z}^{M}\right|=2^{l}$. Every vector $\mathbf{x}=\left[x_{1}, x_{2}, \ldots, x_{M}\right]$ can be represented as

$$
\begin{gathered}
\mathbf{x}=-\frac{1}{2}+\frac{1}{2^{k}} Q+\frac{1}{2^{k+M}} R_{1}+\frac{1}{2^{k+M}} R_{2} \\
+\ldots+\frac{1}{2^{k+j M}} R_{j}+\ldots
\end{gathered}
$$

where $Q \in L / 2^{k} \mathbb{Z}^{M}$ and $R_{j} \in L / 2^{M} L$.

Now, we can construct the lattice version of Scheme I of the previous section. For the data block $\mathbf{x}=\left[x_{1} x_{2} \ldots x_{M}\right]$ $\left(-\frac{1}{2} \leq x_{i}<\frac{1}{2}\right)$, we consider the binary labeling corresponding to $\mathrm{x}$ (as the concatenation of the binary labellings for $Q$, $\left.R_{1}, R_{2}, \ldots\right)$ :

$$
\mathbf{x} \equiv \overline{b_{1} b_{2} \ldots b_{N l} b_{N l+1} \ldots b_{N l+N k} b_{N l+N k+1} \ldots b_{N l+2 N k} \ldots}
$$

Now, we construct the $M$-dimensional transmitted signals $\mathbf{s}_{1}, \mathbf{s}_{2}, \ldots, \mathbf{s}_{N}$ as

$$
\begin{gathered}
\mathbf{s}_{i}=-\frac{1}{2}+\frac{1}{2^{k}} Q_{i}+\frac{1}{2^{k} \alpha^{M}} R_{i 1}+\frac{1}{2^{k} \alpha^{2 M}} R_{i 2} \\
+\ldots+\frac{1}{2^{k+j M}} R_{i j}+\ldots
\end{gathered}
$$

where $Q_{i}$ is a member of $L / 2^{k} \mathbb{Z}^{M}$ (corresponding to label $\overline{b_{i} b_{N+i} . . b_{(l-1) N+i}}$, for $\left.1 \leq i \leq N\right)$ and $R_{i j}$ is a member of $L / 2^{M} L$ (corresponding to label $\overline{b_{l N+(j-1) M N+i} \ldots b_{l N+(j-1) M N+(M-1) N+i}}$, for $1 \leq i \leq N$ and $j=1,2, \ldots)$.

\section{PRACTICAL CONSIDERATIONS AND SIMULATION RESULTS}

Although Scheme II deals with the infinite binary expansion of $x+\frac{1}{2}$ (which is not practically feasible), approximated versions of this mapping can be implemented by lowcomplexity encoders and decoders. A simple approximation of these methods is to split $x+\frac{1}{2}$ as

$$
x+\frac{1}{2}=\overline{0 . b_{1} b_{2} \ldots b_{n}}+r
$$

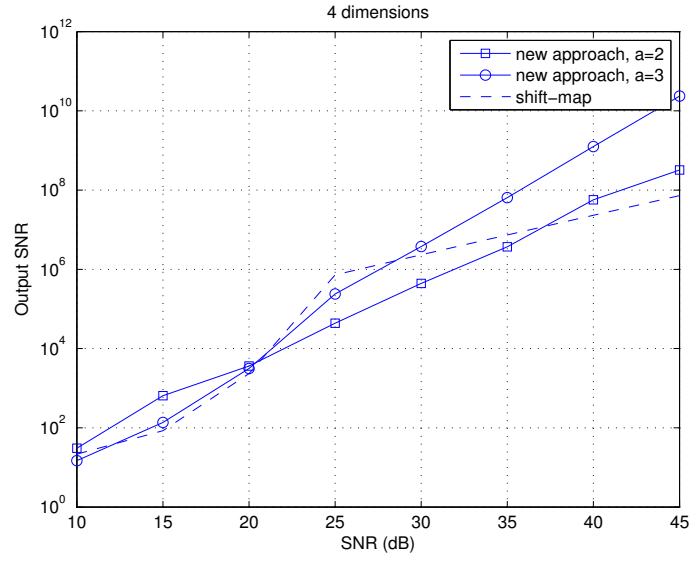

Fig. 2. The output SNR (or SDR) for the first proposed scheme (with $\alpha=4$ and 3) and the shift-map scheme with $a=3$. The bandwidth expansion is $N=4$.

and applying the proposed methods on $\overline{0 . b_{1} b_{2} \ldots b_{n}}$ and send $r$ by a linear mapping over one of the dimensions. For this approximation, the asymptotic slope of the signal to distortion ratio (versus the channel SNR) is 1 (similar to the shiftmap scheme). Indeed, compared to the traditional analog schemes like the shift-map scheme, the new scheme has a gracefull degradation for the low SNR region. At the receiver, decoding can be split among different dimensions without any loss in the performance. Therefore, the complexity of decoding is similar to decoding of scalar quantized data.

In figure 1, for a bandwidth expansion factor of 4 , the performance of Scheme I (with parameters $\alpha=4$ and 3 ) is compared with the shift-map scheme with $a=3$. As we expect, for the shift-map scheme, the signal-todistortion-ratio (SDR) curve saturates at slope 1, while the new scheme offers asymptotic slopes higher than one. For the new scheme, with parameters $\alpha_{1}=4$ and $\alpha_{2}=3$, the asymptotic slope is respectively $\beta_{1}=\frac{4 \log 2}{\log 4}=2$ and $\beta_{2}=\frac{4 \log 2}{\log 3}$ (as expected from Theorem 2). Also, we see that the new scheme provides a gracefull degradation in the low SNR region.

In figure 2, performance of the approximated version of Scheme II (with 5 bits) is compared to the performance of the shift-map scheme for the same setting. Compared to the old method, the new scheme offers a better performance for low SNRs, by using a much simpler decoder.

\section{CONCLUSIONS}

To avoid the mild saturation effect in analog transmission systems and achieving the optimum scaling of the output distortion, we need to use nondifferentiable mappings (more precisely, mappings which are not differentiable on any interval). Two nondifferentiable schemes are introduced in this paper. Both these schemes outperform the traditional analog schemes, in terms of scaling of the output SDR, and one of them almost achieves the optimum SDR scaling with a simple mapping (it asymptotically achieves slope $N$ for 


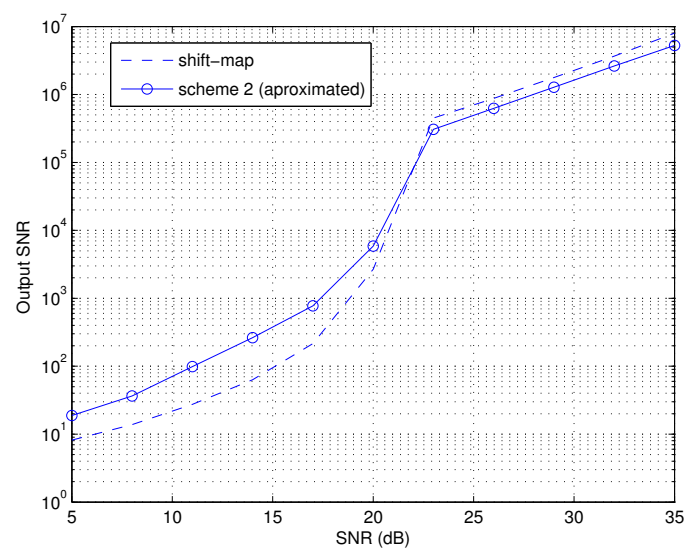

Fig. 3. The output SNR (or SDR) for the approximated version of the proposed scheme II and the shift-map scheme with $a=3$. The bandwidth expansion is $N=4$.

the SDR curve). Also, simulation results show that Piecewise differentiable approximations of these methods offers an acceptable performance (with a low encoding/decoding complexity) for a wide range of channel SNRs.

\section{REFERENCES}

[1] C. Shannon, "A mathematical theory of communication," Bell Syst. Tech. J., 1948

[2] J. Ziv, "Coding theorems for a discrete source with a fidelity criterion," IRE Nat. Conv. Rec., pp. 149-163, Mar. 1959.

[3] E. Ayanoglu and R. M. Gray, "The design of joint source and channel trellis waveform coders," IEEE Trans. Info. Theory, pp. 855-865, Nov. 1987.

[4] N. Farvardin and V. Vaishampayan, "Optimal quantizer design for noisy channels: An approach to combined source-channel coding," IEEE Trans. Info. Theory, pp. 827-838, Nov. 1987.

[5] N. Farvardin, "A study of vector quantization for noisy channels," IEEE Trans. Info. Theory, pp. 799-809, July 1990.

[6] N. Phamdo, N. Farvardin, and T. Moriya, "A unified approach to treestructured and multistage vector quantization for noisy channels," IEEE Trans. Info. Theory, pp. 835-850, May 1993.

[7] M. Wang and T. R. Fischer, "Trellis-coded quantization designed for noisy channels," IEEE Trans. Info. Theory, pp. 1792-1802, Nov. 1994.

[8] B. Chen and G. W. Wornell, "Analog error correcting codes based on chaotic dynamical systems," IEEE Trans. Info. Theory, pp. 1691-1706, July 1998.

[9] V. A. Vaishampayan and I. R. Costa, "Curves on a sphere, shift-map dynamics, and error control for continuous alphabet sources," IEEE Trans. Info. Theory, pp. 1691-1706, July 2003.

[10] I. Rosenhouse and A. J. Weiss, "Combined analog and digital errorcorrecting codes for analog information sources," To appear in IEEE Trans. Communications.

[11] S. Shamai (Shitz), S. Verdu, and R. Zamir, "Systematic lossy source/channel coding," IEEE Trans. Info. Theory, p. 564579, March 1998.

[12] U. Mittal and N. Phamdo, "Hybrid digital-analog (HDA) joint sourcechannel codes for broadcasting and robust communications," IEEE Trans. Info. Theory, pp. 1082-1102, May 2002.

[13] J. Ziv, "The behavior of analog communication systems," IEEE Trans. Info. Theory, pp. 1691-1706, Aug. 1970.

[14] J. H. Conway and N. J. A. Sloane, Sphere packing, lattices and groups. Springer-Verlag, 1999. 